\title{
Characteristics of Oral Microbiota in Patients with Esophageal Cancer in China
}

\author{
Hezi Li, Zhilin Luo, Hong Zhang, Nali Huang, Dong Li, Chengwen Luo, and Tianhu Wang \\ Third Affiliated Hospital of Chongqing Medical University, Chongqing, China \\ Correspondence should be addressed to Tianhu Wang; wtianhu@hospital.cqmu.edu.cn
}

Received 5 September 2021; Revised 4 November 2021; Accepted 15 November 2021; Published 16 December 2021

Academic Editor: Aziz ur Rehman Aziz

Copyright (C) 2021 Hezi Li et al. This is an open access article distributed under the Creative Commons Attribution License, which permits unrestricted use, distribution, and reproduction in any medium, provided the original work is properly cited.

\begin{abstract}
Gut microbiota dysbiosis is closely associated with intestinal carcinogenesis, but the oral microbiota of patients with esophageal squamous cell carcinoma who live in high-risk regions in China has not been fully characterized. In the current study, oral microbial diversity was investigated in 33 patients with esophageal squamous cell carcinoma and 35 healthy controls in Chongqing, China, by sequencing $16 \mathrm{~S}$ rRNA of V3-V4 gene regions. There were statistically significant differences in oral microbiota between esophageal squamous cell carcinoma patients and controls as determined via unweighted pair-group analysis with arithmetic means. At the phylum level, in esophageal squamous cell carcinoma patients, there were comparatively greater amounts of Firmicutes (34.0\% vs. 31.1\%) and Bacteroidetes (25.3\% vs. 24.9\%) and lower amounts of Proteobacteria (17.0\% vs. $20.1 \%)$. At the genus level, esophageal squamous cell carcinoma patients exhibited comparatively greater amounts of Streptococcus (17.3\% vs. $14.5 \%)$ and Prevotella_7 (8.6\% vs. $8.5 \%)$ and lower amounts of Neisseria (8.1\% vs. 10.7\%). Using a linear discriminant analysis effect size method, Planctomycetes and Verrucomicrobia were identified in the esophageal squamous cell carcinoma group. 10 genera were higher abundances identified in the healthy control group, and different 10 genera were identified in the esophageal squamous cell carcinoma group. In the present study, there were significant differences in oral microbial compositions of esophageal squamous cell carcinoma patients and healthy controls. Further longitudinal and mechanistic studies are needed to further characterize relationships between oral microbiota and esophageal squamous cell carcinoma.
\end{abstract}

\section{Introduction}

Esophageal cancer is one of the most common cancers of the upper gastrointestinal tract, and it is currently the ninth most common cancer overall and the sixth highest cause of cancer deaths globally [1]. Esophageal cancer is more common in males [2].

Esophageal squamous cell carcinoma is more common in Asians, whereas esophageal adenocarcinoma is more common in Europeans [3]. The prevalence of esophageal cancer has increased in recent years but due to a shortage of relevant biomarkers, it is usually not diagnosed early, and there are still few biomarkers to utilize when devising treatment for patients with advanced-stage esophageal cancer. The present study was focused on microbes that may be useful for the tentative diagnosis or early detection of esophageal cancer.
Recent investigations have resulted in the discovery of associations between the oral microbiota and oral diseases, including systemic diseases and cancers $[4,5]$. It has been suggested that this is because the oral microbiota plays a key role in aspects of the human immune system, and the immune-inflammatory response of the host to components of the oral microbiome may lead to inflammation [6]. The oral microbiota is almost the same as the esophageal microbiota, and alterations to the oral microbiome may directly affect the esophagus [7]. The upper gastrointestinal tract microbiome and the abundance of bacteria in saliva reportedly only fluctuate slightly at certain time points and then return to their original level during the day $[8,9]$.

The ongoing development of DNA sequencing technologies and dimensional reduction of algorithm development have made microbiome research cheaper and more controllable. Numerous online microbiome databases have now 
been established via cross-sectional and longitudinal studies, and they are updated constantly [10]. Notably, however, there are still a lot of unknown factors pertaining to esophageal cancer, particularly with regard to associations with the oral microbiome. The present study was conducted to investigate relationships between the oral microbiome and esophageal cancer in Asians and to generate a corresponding oral microbiome database. Saliva samples were collected, and differences in salivary microbiomes between esophageal cancer patients and controls were investigated via $16 \mathrm{~S}$ rDNA sequencing.

\section{Materials and Methods}

2.1. Study Patients and Specimen Collection. The current case-control study included 33 patients who were diagnosed with esophageal squamous cell carcinoma and underwent upper gastrointestinal examination at the Third Affiliated Hospital of Chongqing Medical University in Chongqing, China, between July 2019 and September 2020. The control group was composed of 35 people who underwent medical examinations at a local hospital. Subjects in the control group had no history of esophagitis or esophagectomy and had not taken antibiotics and proton pump inhibitors in the last month. Patients with rheumatoid arthritis, coronary heart disease, hyperlipidemia, or periodontal conditions were excluded from the study [11]. All the samples were collected during 8:30 a.m. to 11:30 a.m., and the participants were asked to rinse their mouths with flowing water to eliminate food debris and then waited natural salivary secretion. Further details of the exclusion criteria applied are shown in Table 1. Mean and standard deviation are used to describe characteristics of continuous variables except for the age variable. Quartile are used for the age factor. Frequency (percentage) and constituent rations deviation are used to describe characteristics of categorical variable. $T$ test or chi-square test are used to compare variables between the two groups. Participants were asked to spit into a sterile tube with cryopreservation with at least $4 \mathrm{ml}$ in $10 \mathrm{~min}$, and they had no oral disease. Saliva samples were collected from all participants and immediately frozen at $-80^{\circ} \mathrm{C}$ using sterile tubes. There were no significant differences in age, gender, or body mass index between the two groups $(p>0.05)$.

2.2. Microbial $16 S$ rDNA Sequencing. DNA was extracted from saliva using the FastDNA Spin Kit for Soil (MP Biomedicals, USA) in accordance with manufacturer's instructions, then NanoDrop2000 was used for quality control. Gene amplification was performed with an ABI GeneAmp 9700 instrument. Replicate PCR reactions were performed using modified universal bacterial primers designed to amplify the V3-V4 16S rDNA gene region [12]; 338F $\left(5^{\prime}\right.$ ACTCCTACGGGAGGCAGCAG- $\left.3^{\prime}\right)$ and 806R ( $5^{\prime}$-GGAC TACHVGGGTWTCTAAT- $\left.3^{\prime}\right)$. The cycling conditions were $95^{\circ} \mathrm{C}$ for $3 \mathrm{~min}$, followed by twenty-seven cycles of $95^{\circ} \mathrm{C}$ for $30 \mathrm{~s}, 55^{\circ} \mathrm{C}$ for $30 \mathrm{~s}$, and $72^{\circ} \mathrm{C}$ for $45 \mathrm{~s}$, then a final elongation step of $72^{\circ} \mathrm{C}$ for $10 \mathrm{~min}$ [13]. Every sample were tested in duplicate of three times. PCR products for each sample were detected by $2 \%$ agarose gel electrophoresis, then the products were recovered by the AxyPrep DNA Gel Extraction Kit. PCR products were quantified by the Quantus TM Fluorometer. In order to construct library, using PCR to ligation of Illumina barcodes and adaptors. The completed library was sequenced via an Illumina Miseq 2X300bp platform in accordance with Illumina's recommended protocol.

2.3. Statistical Analysis. After $16 \mathrm{~S}$ rDNA sequencing quality control, the length was using a threshold of $50 \mathrm{bp}$ and filtered lower abundance raw reads. Operational taxonomic units (OTUs) were set to measure microbial abundance according to the $16 \mathrm{~S}$ rDNA conservation sequence. The Silva database (http://www.arb-silva.de) were used to classify the bacteria [14]. A Bayesian algorithm was used to identify the species composition of samples, including microbial phylum, class, order, family, and genus. Prior to diversity comparisons, species accumulation curves were used to assess sample size and species richness.

QIIME software was used to calculate the alpha diversity index, including the observed species index, Chao index, Shannon index, and Simpson index. These indices were used to assess evenness and abundance within groups based on alpha diversity. Rarefaction curve analysis was used to identify species richness and to assess sequence data plausibility. Beta diversity was calculated using weighted and unweighted UniFrac analysis estimated by distances between samples from esophageal squamous cell carcinoma patients and controls. A beta diversity method with principal component analysis, principal coordinates analysis, and unweighted pair-group method with an arithmetic means were used to determine the different oral microbiota in the two groups. Analysis of similarities and Adonis were then used to assess the statistical significance of differences between the esophageal squamous cell carcinoma group and the control group.

The Wilcoxon rank-sum test and phylogenetic investigation of communities were used to identify distinct microbial taxa in the esophageal squamous cell carcinoma group and the control group. Linear discriminant analysis effect size was used to identify taxa that differed in the esophageal squamous cell carcinoma group and the control group, via the nonparametric factorial Kruskal-Wallis sum-rank test function in $\mathrm{R}$ (version 3.4.3) [15].

\section{Results}

3.1. Characteristics of Subjects and Oral Microbial Composition. A total of 33 patients with esophageal cancer (28 men and 5 women, 56-76 years of age) and 35 agematched were included in the final analysis after quality control of all samples. All of the subjects in the study were Asian. There was no significant difference in dietary habits between the esophageal squamous cell carcinoma and control groups. Considering the sample size, smoking stratified according to duration, and drinking stratified by alcohol consumption stratification. The smoking ratio was no significant differences between the esophageal squamous cell carcinoma group and control group $(p=0.319)$, the drinking ratio was significant differences between the two groups 
TABLE 1: General demographic characteristics of the esophageal cancer group and the control group.

\begin{tabular}{|c|c|c|c|c|}
\hline Variable & Control & ESCC patients & $t / \chi^{2}$ value & $p$ \\
\hline Age* $^{*}$ & $61.00(55.00 \sim 70.00)$ & $66.00(56.00 \sim 68.50)$ & $554.00-$ & 0.773 \\
\hline Gender $(n / \%)$ & & & 3.32 & 0.069 \\
\hline Female & $12(34.29)$ & $5(15.15)$ & & \\
\hline Male & $23(65.71)$ & $28(84.85)$ & & \\
\hline $\mathrm{BMI}($ mean $\pm \mathrm{SD})$ & $23.8 \pm 2.0$ & $22.7 \pm 2.8$ & 1.84 & 0.070 \\
\hline Smoking $(n / \%)$ & & & 0.992 & 0.319 \\
\hline$<35$ years & $18(51.43)$ & $13(37.14)$ & & \\
\hline$\geq 35$ years & $17(48.57)$ & $20(62.86)$ & & \\
\hline Drinking $(n / \%)$ & & & 5.842 & $<0.05$ \\
\hline$<100 \mathrm{ml} /$ day & $25(71.43)$ & $14(42.42)$ & & \\
\hline$\geq 100 \mathrm{ml} /$ day & $10(28.57)$ & $19(57.58)$ & & \\
\hline Frequency of eating spicy food $(n / \%)$ & & & 4.37 & 0.358 \\
\hline Never & $7(20.00)$ & $3(9.09)$ & & \\
\hline 1-3 times/month & $6(17.14)$ & $5(15.15)$ & & \\
\hline 1-2 times/week & $8(22.86)$ & $4(12.12)$ & & \\
\hline 3-5 times/week & $6(17.14)$ & $9(27.27)$ & & \\
\hline Every day & $8(22.86)$ & $12(36.36)$ & & \\
\hline Missing teeth $(n / \%)$ & & & 6.77 & 0.148 \\
\hline None & $19(54.29)$ & $12(36.36)$ & & \\
\hline $1-4$ & $11(31.43)$ & $12(36.36)$ & & \\
\hline $5-8$ & $2(5.71)$ & $2(6.06)$ & & \\
\hline $9-11$ & $3(8.57)$ & $2(6.06)$ & & \\
\hline$\geq 12$ & $0(0.00)$ & $5(15.15)$ & & \\
\hline Frequency of brushing teeth $(n / \%)$ & & & 5.17 & 0.075 \\
\hline Never & $0(0.00)$ & $3(9.09)$ & & \\
\hline 1 time/day & $20(57.14)$ & $22(66.67)$ & & \\
\hline$\geq 2$ times/day & $15(42.86)$ & $8(24.24)$ & & \\
\hline
\end{tabular}

Note: ${ }^{*} M$ (P25 P75); ESCC: esophageal squamous cell carcinoma; ${ }^{*} p<0.05$.

$(p<0.05)$. With regard to oral condition, the control group reported more toothbrushing and exhibited fewer decayed teeth than the esophageal squamous cell carcinoma group, but these differences were not statistically significant.

All salivary samples were sequenced to evaluate oral bacterial diversity in the esophageal squamous cell carcinoma group and the control group. There were different 128 OTUs taxa and different 42 OTUs taxa in esophageal squamous cell carcinoma group and control group, respectively. Twenty phyla and 275 genera were identified, and there were no significant differences in alpha diversity between the patient group and the control group. Shannon index was 3.86 and 3.91 in the esophageal squamous cell carcinoma patients and control group, respectively $(p=0.61$; Figure $1(\mathrm{a}))$. Simpson index was 0.053 and 0.049 in the esophageal squamous cell carcinoma patients and control group, respectively $(p=0.62$; Figure $1(\mathrm{~b}))$. The $p$ value of observed OTUs was 0.73 .

3.2. Differences between the Esophageal Cancer Group and the Control Group. In beta diversity analyses, there was a statistically significant difference between the control group and the esophageal squamous cell carcinoma group as determined by the unweighted pair-group method with an arithmetic means. There was no statistically significance difference in clustering between the two groups as determined by the weighted pair-group method with an arithmetic means. The different oral microbiota was identified between esophageal squamous cell carcinoma patients and control groups according to permutational multivariate analysis of variance (Bray-Curtis $p=0.031$; unweighted $p=$ 0.001 ; weighted $p=0.28$ ) and analysis of similarities (BrayCurtis $p=0.048$; unweighted $p=0.004$; weighted $p=0.177$ ). These results indicated comparative differences in the abundance of microbiota rather than differences in the types of bacteria in the saliva of the esophageal squamous cell carcinoma group and the control group (Figure 2). At the phylum level, Firmicutes $(34.0 \%$ vs. $31.1 \% ; p=0.17$ ) and Bacteroidetes $(25.3 \%$ vs. $24.9 \%$; $p=0.63)$ were more abundant in the esophageal squamous cell carcinoma group than in the control group, whereas Proteobacteria (17.0\% vs. 20.1\%; $p=0.43$ ) were less abundant. At the genus level, Streptococcus $(17.3 \%$ vs. $14.5 \% ; p=0.10)$ and Prevotella_7 (8.6\% vs. $8.5 \% ; p=0.85)$ was more abundant in the esophageal 


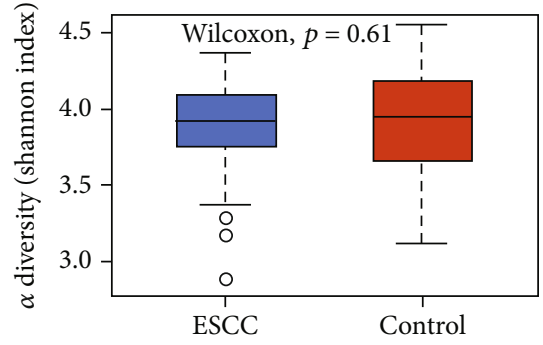

(a)

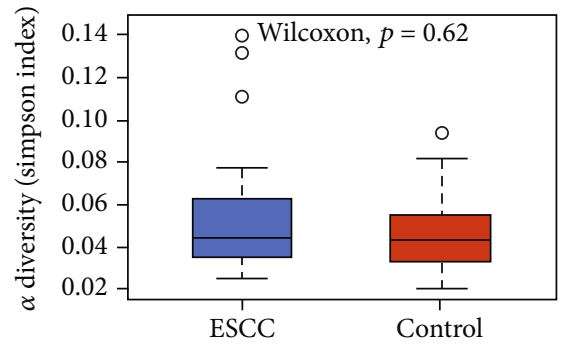

(b)

FIgURE 1: Alpha diversity and richness of oral microbiota in the esophageal squamous cell carcinoma group and the control group determined via the Wilcoxon test. There were no significant differences in alpha diversity between the esophageal squamous cell carcinoma and control groups. (a) Shannon index $(p=0.61)$. (b) Simpson index $(p=0.62)$.

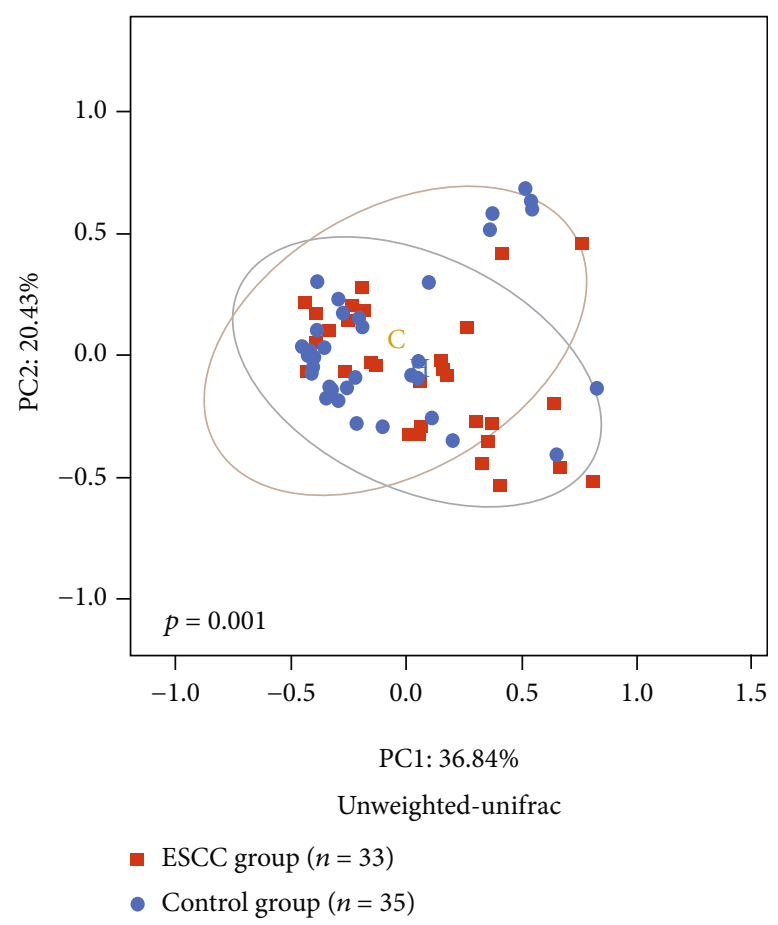

(a)

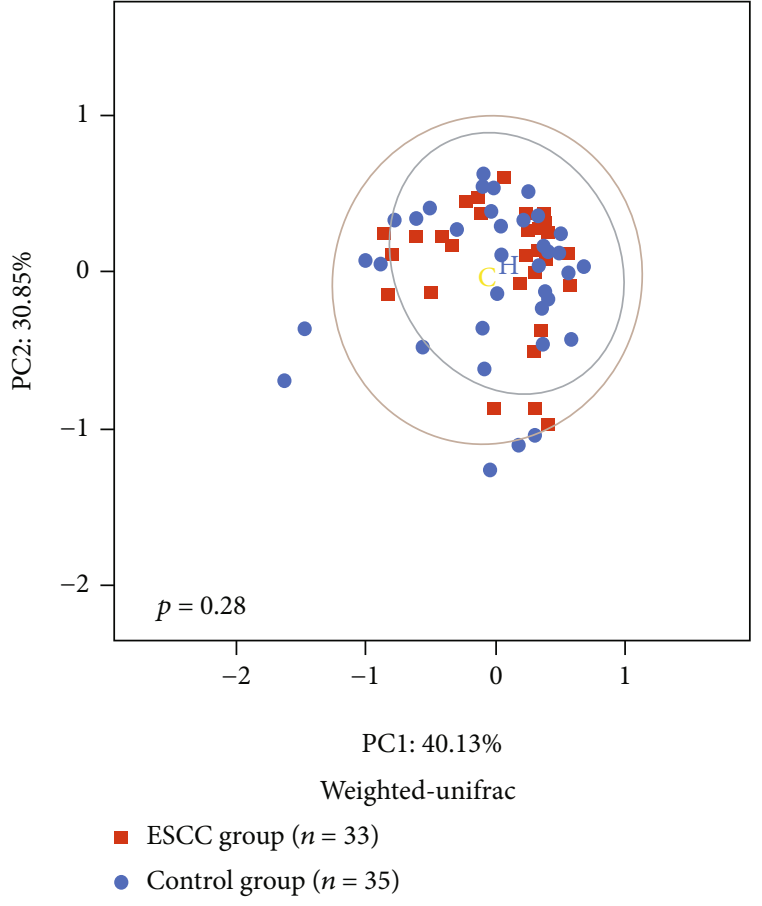

(b)

Figure 2: Beta diversity of oral microbiota in the esophageal squamous cell carcinoma group and the control group. There were significant differences in beta diversity between the esophageal squamous cell carcinoma and control groups. (a) Principal coordinate analysis using unweighted-UniFrac of beta diversity. (b) Principal coordinate analysis using weighted-UniFrac of beta diversity.

squamous cell carcinoma group, but Neisseria ( $8.1 \%$ vs. $10.7 \%$; $p=0.22$ ) were less abundant (Figure 3). In Mann-Whitney $U$ testing to identify differences in bacteria between the two groups, at the phylum level, Planctomycetes and Verrucomicrobia were identified in the esophageal squamous cell carcinoma group but not in the control group $(p<0.05$; Figure 4(a)). At the genus level, Capnocytophaga were predominant detected in the esophageal squamous cell carcinoma group, and at the family level, Lachnospiraceae were predominant detected in the control group ( $p<0.05$; Figure 4(b)).

Based on clade abundances at all taxonomic levels, the linear discriminant analysis effect size system for biomarker discovery was used to identify statistically significant biomarkers in the saliva of the two groups. There were 10 gen- era that were identified in the healthy control group, and different 10 were identified in the esophageal squamous cell carcinoma group (Figure 5). Four phylum-level greater of relative abundance were identified in the esophageal squamous cell carcinoma group, Bacteroidetes, Firmicutes, Fusobacteria, and Actinobacteria $(p<0.05$, linear discriminant analysis $>$ 2 ). Three phylum-level greater of relative abundance were identified in the control group, Firmicutes, Actinobacteria, and Proteobacteria $(p<0.05$, linear discriminant analysis $>$ 2). Capnocytophaga were significantly more predominant in the esophageal squamous cell carcinoma group than in the control group, and conversely, Lachnospiraceae were significantly more predominant in the control group than in the esophageal squamous cell carcinoma group. 


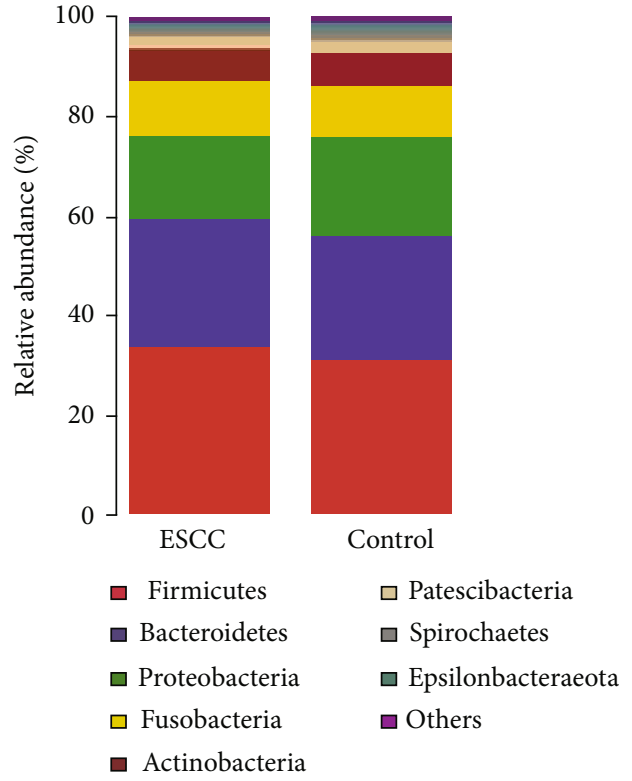

(a)

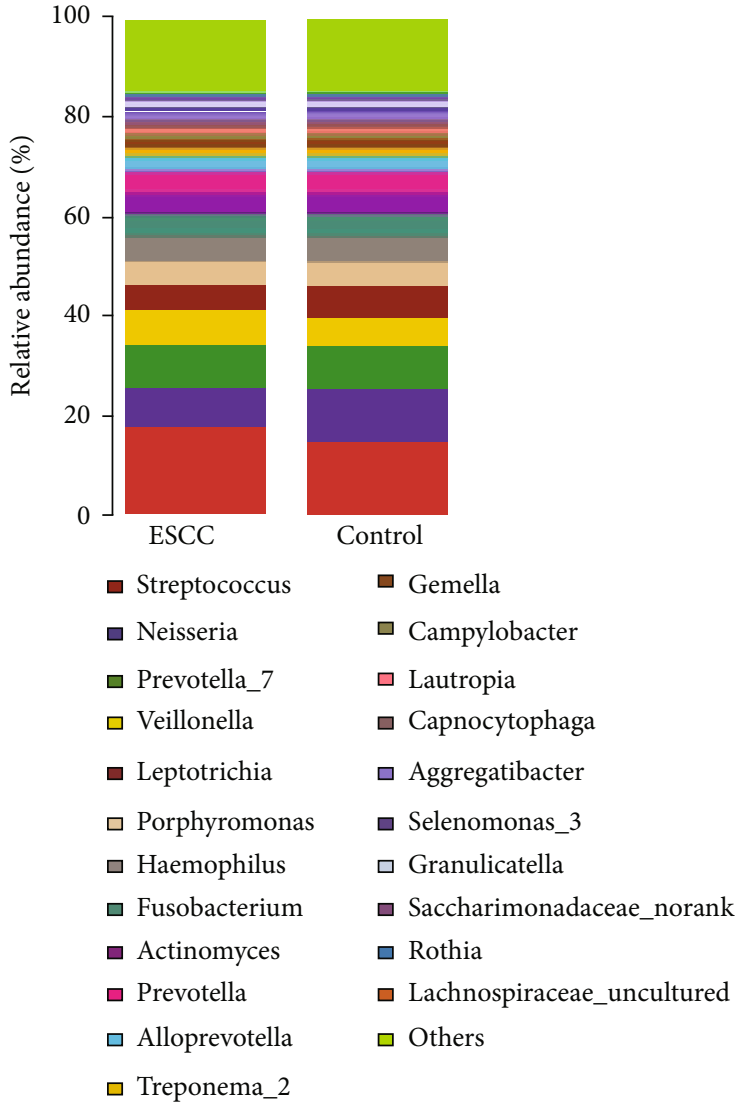

(b)

Figure 3: Oral microbial relative abundances at phylum and genus level between the esophageal squamous cell carcinoma and control groups. Identified 8 phylum and 22 genera in two groups, respectively. (a) Phylum level. (b) Genus level.

\section{Discussion}

Attention to the oral microbiome is increasing in terms of its functions as a predictor and biomarker in human cancers. In other studies investigating the gastrointestinal tract, there have been associations between oral microbiome parameters and digestive tract cancers [16-18]. All subjects in the current study had good dentition and were recorded the number of missing teeth, which has seldom been considered in previous studies investigating the oral microbiota and esophagus microbiome. Short-term dietary intake does not influence salivary microbiome; moreover, the salivary microbiome of individuals does not alter during a day [19, 20]. Some recent studies reported oral microbiota have a linkage to autoimmune diseases, and some associated with cancer, and these findings established a risk prediction model through collected oral microbiome for ESCC [21, 22]. In the current study, there were no statistically significant differences in alpha diversity between the patient group and the control group, but there was a statistically significance difference in relative abundances as determined via beta diversity. Therefore, the oral microbiome may play a key role in the development of esophageal squamous cell carcinoma.

In the present study, OTU phylogeny was compared in saliva samples from the two groups, and the most abundant OTU phyla in the esophageal squamous cell carcinoma and control groups, respectively, were Firmicutes (34.0\% vs. $31.10 \%)$, Bacteroidetes $(25.3 \%$ vs. $24.9 \%)$, Proteobacteria (17.0\% vs. $20.1 \%$ ), and Fusobacteria (10.9\% vs. $10.3 \%)$. The greater relative abundance of Firmicutes and lower relative abundance of Proteobacteria in the esophageal cancer group are concordant with Snider et al. [23]. Notably however, Snider et al. [24] reported contrasting results in esophageal adenocarcinoma patients at the phylum level, and their results indicated lower diversity in esophageal adenocarcinoma. They also reported significant differences in microbiomes between patients with Barrett's esophagus and patients with esophageal adenocarcinoma. In Zhao et al. [25], the oral microbiota of 31 esophageal cancer patients in China exhibited more Firmicutes and less Proteobacteria than controls, which is consistent with the current study. Their findings are that the most significantly increased taxa were six species, while the most significantly decreased taxa were five species. And they concluded that Prevotella may be associated with esophageal cancer development.

At the genus level, the most abundant OTUs in saliva samples in the esophageal squamous cell carcinoma group and the control group, respectively, were Streptococcus (17.3\% vs. $14.5 \%)$, Neisseria ( $8.1 \%$ vs. $10.7 \%)$, and Prevotella_7 (8.6\% vs. $8.5 \%)$. In the esophageal squamous cell carcinoma group, there was a greater relative abundance of Streptococcus and a lower relative abundance of Neisseria. 


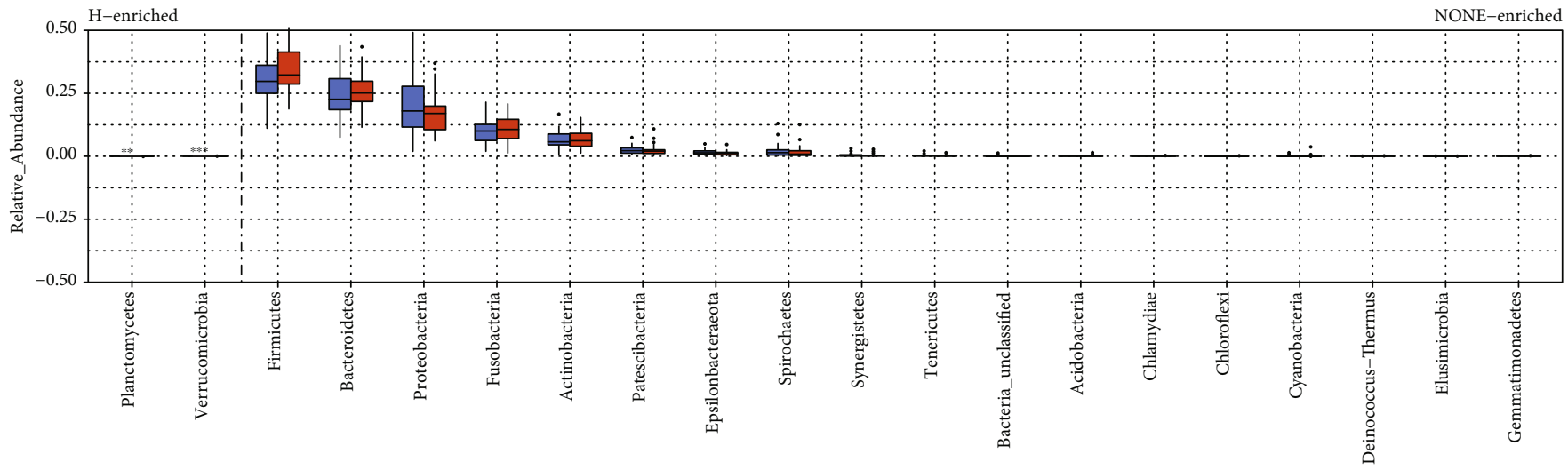

(a)

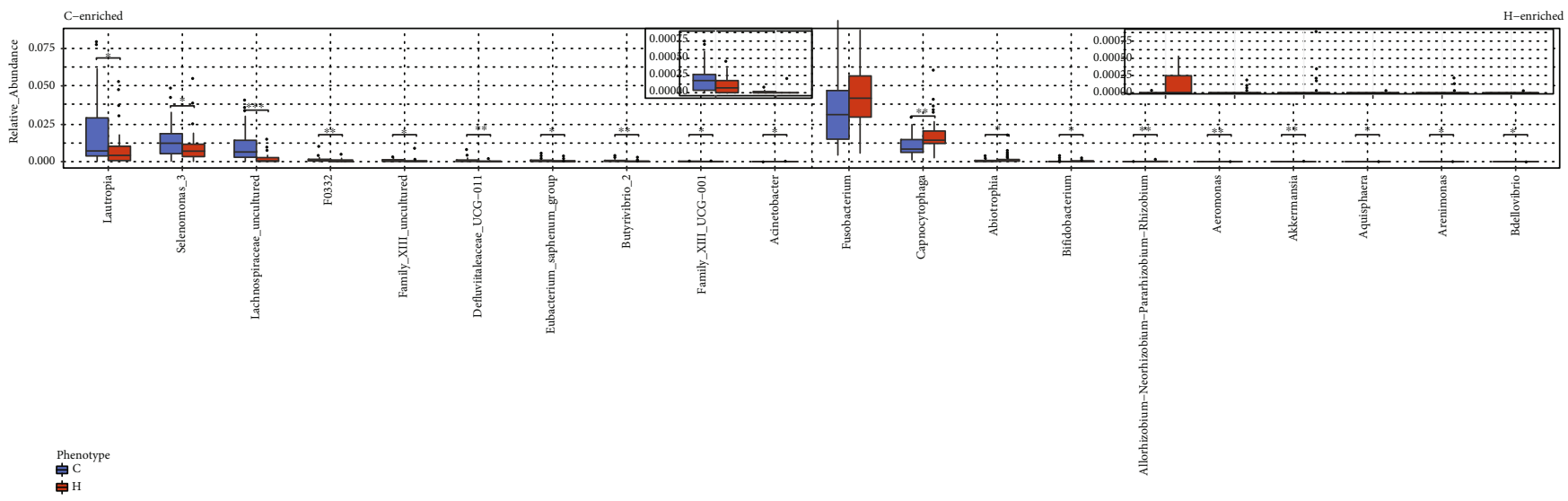

(b)

FIGURE 4: Boxplot representing community differences between the esophageal cancer group and the control group determined via the Wilcoxon test. (a) Phylum level. (b) Genus level. ${ }^{* *} p<0.01,{ }^{* * *} p<0.001$.

In previous studies, Streptococcus in the esophagus have been classified into two types, the dominant taxon within the healthy esophagus was Streptococcus, and the esophageal adenocarcinoma cascade was reportedly characterized by a shift towards a dominance of Gram-negative bacterial species $([26,27])$. In another study in Chinese Asians, esophageal squamous cell carcinoma patients exhibited less Streptococcus at the genus level [28], which differs from the results of the present study at the genus level, but the results were concordant at the phylum level, may be due to the different study designs, sample origins and characteristics of local oral microbiota. Some studies have reported enrichment of specific oral bacterial species such as Fusobacterium nucleatum [29], but other studies have reported conflicting results [30]. In one study, protective species were closely connected among different phyla; for example, lower Neisseria was associated with higher esophageal squamous cell carcinoma risk [31], which is consistent with the current study. Therefore, the oral microbiome may play a key role in the development of esophageal cancer.

Interestingly, Planctomycetes and Verrucomicrobia were only in the esophageal squamous cell carcinoma group, and this two phylum microbiomes were core microbiomes. However, the relative of this two phylum microbiomes abundance were low; Planctomycetes and Verrucomicrobia may be a potential predictor of esophageal squamous cell carcinoma, so there still need some experimental validation. In the present study, Capnocytophaga were significantly more prevalent in the esophageal squamous cell carcinoma group, and Lachnospiraceae was significantly more prevalent in the control group. Capnocytophaga may be a pathogenic bacterium in oral cavity squamous cell cancer [32, 33], and in one case report, Capnocytophaga invaded the hyperplasia of an immunocompromised patient [34]. In these studies, a higher abundance of Capnocytophaga in the oral cavity was associated with oral diseases, and it may be a promising biomarker for predicting esophageal squamous cell carcinoma. In a previous study, Lachnospiraceae functioned as a shortchain fatty acid producer [35], and this family of anaerobic bacteria is reportedly found at relatively low levels in colorectal cancer patients $[16,36]$. This result is consistent with the present study, in which there was a higher abundance of Lachnospiraceae in healthy individuals. There was a hypothesis indicating that the microbiome is dynamic 


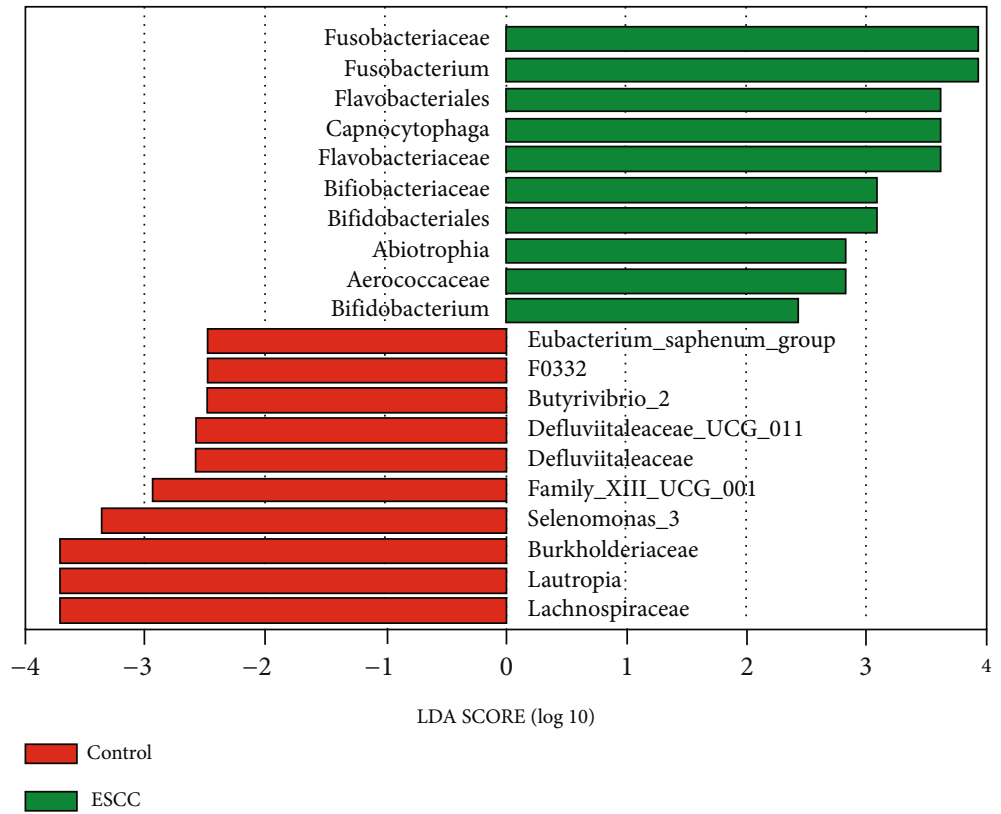

Figure 5: Significant differences in oral microbial taxa between the esophageal squamous cell carcinoma group and the control group. Identified 10 genera in two groups, respectively. Threshold linear discriminant analysis (linear discriminant analysis $>2, p<0.05$ ).

[37]. Oral microbiome changes in esophageal squamous cell carcinoma patients, whereas oral microbiome remains unchanged in control group. Therefore, the current study indicated that differences in oral microbiota in the current study provide evidence in support of distinctions between esophageal squamous cell carcinoma patients and controls at the genus level and differences in overall ecological structure.

Strengths of the current study are that all patients included were esophageal squamous cell carcinoma; there was no other pathological types of ecological cancer. In order to minimize confounding variables of the microbiome, we designed a rigorous study and followed strict collecting samples standards with health oral cavity. The case-control groups have the same characteristics, such as age, gender, race, and smoking and drinking conditions. These potential variables which may cause confounding bias can be avoided by having the same characteristics between the two groups. Our sample size was relatively large, and clinical studies are still important, and its findings provide hypothesis for following metabolism and microbiome function studies. Limitations of current study are that it describes the different oral microbiome relationship associated with the risk of esophageal squamous cell carcinoma, but further prospective longitudinal studies related to esophageal squamous cell carcinoma development and progression are needed to establish causation. Although models that humanizing immune system are improving, microbial models are still different from human body.

Therefore, multicenter studies are needed in future studies.

\section{Conclusion}

The current study identified direct relationships between esophageal cancer and different oral microbiota, and the functions of the different oral microbiomes were predicted via the construction of a database. The results indicate that differences in the oral microbiota may have causative effects on the risk of esophageal squamous cell carcinoma at phylum level. In addition, the oral microbiota abundance is different between esophageal squamous cell carcinoma and control group at the genus level. Future studies and more mechanistic studies are required in order to control confounding variables.

\section{Data Availability}

The raw data in this study are publicly available at https:// submit.ncbi.nlm.nih.gov/subs/bioproject/.

\section{Ethical Approval}

Demographics, clinical data, and questionnaire were collected. The study ethics was approved by the Third Affiliated Hospital of Chongqing Medical University institutional review board and the number of ethics review is 2019 (4).

\section{Consent}

All participants provided written informed consent.

\section{Conflicts of Interest}

The authors declare no potential conflicts of interest with respect to the authorship or publication of this article.

\section{Authors' Contributions}

Hezi Li participated in the design of this study and drafted this manuscript. Zhilin Luo finished literature search and approved manuscript editing. Hong Zhang collected the patients samples and clinical details. Nanli Huang, 
Chengwen Luo, and Dong Li participated in performing measurements and the data analysis. Tianhu Wang participated design of this study and carried out the final review.

\section{Acknowledgments}

This research was supported by the Wu Jieping Medical Foundation (320.6750.19077).

\section{References}

[1] F. Bray, J. Ferlay, I. Soerjomataram, R. L. Siegel, L. A. Torre, and A. Jemal, "Global cancer statistics 2018: GLOBOCAN estimates of incidence and mortality worldwide for 36 cancers in 185 countries," CA: A Cancer Journal for Clinicians, vol. 68, no. 6, pp. 394-424, 2018.

[2] S.-H. Xie and J. Lagergren, "The male predominance in esophageal adenocarcinoma," Clinical Gastroenterology and Hepatology, vol. 14, no. 3, pp. 338-347.e1, 2016.

[3] H. G. Coleman, S.-H. Xie, and J. Lagergren, "The epidemiology of esophageal adenocarcinoma," Gastroenterology, vol. 154, no. 2, pp. 390-405, 2018.

[4] J. Sun and P. K. Dudeja, Eds., Mechanisms UnderlyingHostMicrobiome Interactions in Pathophysiology of Human Diseases, Springer, Boston, MA, USA, 2018.

[5] J. Yang, X. Mu, Y. Wang et al., "Dysbiosis of the salivary microbiome is associated with non-smoking female lung cancer and correlated with immunocytochemistry markers," Frontiers in Oncology, vol. 8, p. 520, 2018.

[6] D. T. Graves, J. D. Corrêa, and T. A. Silva, "The oral microbiota is modified by systemic diseases," Journal of Dental Research, vol. 98, no. 2, pp. 148-156, 2019.

[7] R. Vasapolli, K. Schütte, C. Schulz et al., "Analysis of transcriptionally active bacteria throughout the gastrointestinal tract of healthy individuals," Gastroenterology, vol. 157, no. 4, pp. 1081-1092.e3, 2019.

[8] T. S. Schmidt, M. R. Hayward, L. P. Coelho et al., "Extensive transmission of microbes along the gastrointestinal tract," vol. 8, Article ID e42693, 2019.

[9] J. Wang, Z. Jia, B. Zhang, L. Peng, and F. Zhao, "Tracing the accumulation of in vivo human oral microbiota elucidates microbial community dynamics at the gateway to the GI tract," Gut, vol. 69, no. 7, pp. 1355-1356, 2020.

[10] P. Xian, Z. Xuedong, X. Xin et al., "The oral microbiome bank of China," International Journal of Oral Science, vol. 10, no. 2, p. 16, 2018.

[11] J. Lv, L. Guo, J.-J. Liu, H.-P. Zhao, J. Zhang, and J.-H. Wang, "Alteration of the esophageal microbiota in Barrett's esophagus and esophageal adenocarcinoma," WJG, vol. 25, no. 18, pp. 2149-2161, 2019.

[12] N. P. Deshpande, S. M. Riordan, N. Castaño-Rodríguez, M. R. Wilkins, and N. O. Kaakoush, "Signatures within the esophageal microbiome are associated with host genetics, age, and disease," Microbiome, vol. 6, no. 1, p. 227, 2018.

[13] D. Nasrollahzadeh, R. Malekzadeh, A. Ploner et al., "Variations of gastric corpus microbiota are associated with early esophageal squamous cell carcinoma and squamous dysplasia," Scientific Reports, vol. 5, no. 1, p. 8820, 2015.

[14] M. May and J. A. Abrams, "Emerging insights into the esophageal microbiome," Curr Treat Options Gastro, vol. 16, no. 1, pp. 72-85, 2018.
[15] Y. Tong, L. Zheng, P. Qing et al., "Oral microbiota perturbations are linked to high risk for rheumatoid arthritis," Frontiers in Cellular and Infection Microbiology, vol. 9, p. 475, 2020.

[16] B. Flemer, R. D. Warren, M. P. Barrett et al., "The oral microbiota in colorectal cancer is distinctive and predictive," Gut, vol. 67 , no. 8, pp. 1454-1463, 2018.

[17] S. Kageyama, T. Takeshita, K. Takeuchi et al., "Characteristics of the salivary microbiota in patients with various digestive tract cancers," Frontiers in Microbiology, vol. 10, p. 1780, 2019.

[18] G. Yu, J. Torres, N. Hu et al., "Molecular characterization of the human stomach microbiota in gastric cancer patients," Frontiers in Cellular and Infection Microbiology, vol. 7, p. 302, 2017.

[19] D. Belstrøm, P. Holmstrup, A. Bardow, A. Kokaras, N.E. Fiehn, and B. J. Paster, "Temporal stability of the salivary microbiota in oral health," PLoS One, vol. 11, no. 1, article e0147472, 2016.

[20] J. Kim, M. Lee, B. Baldwin-Hunter et al., "Minimal associations between short-term dietary intake and salivary microbiome composition," Microorganisms, vol. 9, no. 8, p. 1739, 2021.

[21] F. Liu, M. Liu, Y. Liu et al., "Oral microbiome and risk of malignant esophageal lesions in a high-risk area of China: a nested case-control study," Chinese Journal of Cancer Research, vol. 32, no. 6, pp. 742-754, 2020.

[22] M. Liu, Z. Liu, H. Cai et al., "A model to identify individuals at high risk for esophageal squamous cell carcinoma and precancerous lesions in regions of high prevalence in China," Clinical Gastroenterology and Hepatology, vol. 15, no. 10, pp. 15381546.e7, 2017.

[23] E. J. Snider, G. Compres, D. E. Freedberg et al., "Barrett's esophagus is associated with a distinct oral microbiome," Gastroenterology, vol. 9, no. 3, article e135, 2018.

[24] E. J. Snider, G. Compres, D. E. Freedberg et al., "Alterations to the esophageal microbiome associated with progression from Barrett's esophagus to esophageal adenocarcinoma," Cancer Epidemiology, Biomarkers \& Prevention, vol. 28, no. 10, pp. 1687-1693, 2019.

[25] Q. Zhao, T. Yang, Y. Yan et al., "Alterations of oral microbiota in Chinese patients with esophageal cancer," Frontiers in Cellular and Infection Microbiology, vol. 10, article 541144, 2020.

[26] Z. Pei, E. J. Bini, L. Yang, M. Zhou, F. Francois, and M. J. Blaser, "Bacterial biota in the human distal esophagus," Proceedings of the National Academy of Sciences, vol. 101, no. 12, pp. 4250-4255, 2004.

[27] L. Yang, X. Lu, C. W. Nossa, F. Francois, R. M. Peek, and Z. Pei, "Inflammation and intestinal metaplasia of the distal esophagus are associated with alterations in the microbiome," Gastroenterology, vol. 137, no. 2, pp. 588-597, 2009.

[28] D. Shao, E. Vogtmann, A. Liu et al., "Microbial characterization of esophageal squamous cell carcinoma and gastric cardia adenocarcinoma from a high-risk region of China," Cancer, vol. 125, no. 22, pp. 3993-4002, 2019.

[29] K. Yamamura, Y. Baba, S. Nakagawa et al., "Human microbiome fusobacterium nucleatum in esophageal cancer tissue is associated with prognosis," Clinical Cancer Research, vol. 22, no. 22, pp. 5574-5581, 2016.

[30] I. Amir, F. M. Konikoff, M. Oppenheim, U. Gophna, and E. E. Half, "Gastric microbiota is altered in oesophagitis and Barrett's oesophagus and further modified by proton pump 
inhibitors," Environmental Microbiology, vol. 16, no. 9, pp. 2905-2914, 2014.

[31] B. A. Peters, J. Wu, Z. Pei et al., "Oral microbiome composition reflects prospective risk for esophageal cancers," Cancer Research, vol. 77, no. 23, pp. 6777-6787, 2017.

[32] I. Ganly, L. Yang, R. A. Giese et al., "Periodontal pathogens are a risk factor of oral cavity squamous cell carcinoma, independent of tobacco and alcohol and human papillomavirus," International Journal of Cancer, vol. 145, no. 3, pp. 775-784, 2019.

[33] M. Perera, N. N. al-hebshi, I. Perera et al., "Inflammatory bacteriome and oral squamous cell carcinoma," Journal of Dental Research, vol. 97, no. 6, pp. 725-732, 2018.

[34] D. N. Fredricks, M. M. Schubert, and D. Myerson, "Molecular identification of an invasive gingival bacterial community," Clinical Infectious Diseases, vol. 41, no. 1, pp. e1-e4, 2005.

[35] Z. Zhang, H. Cao, N. Song, L. Zhang, Y. Cao, and J. Tai, “Longterm hexavalent chromium exposure facilitates colorectal cancer in mice associated with changes in gut microbiota composition," Food and Chemical Toxicology, vol. 138, article 111237, 2020.

[36] D. Sun, R. Bai, W. Zhou et al., “Angiogenin maintains gut microbe homeostasis by balancing $\alpha$-Proteobacteria and Lachnospiraceae," Gut, vol. 70, no. 4, pp. 666-676, 2021.

[37] B. T. Rosier, P. D. Marsh, and A. Mira, "Resilience of the oral microbiota in health: mechanisms that prevent dysbiosis," Journal of Dental Research, vol. 97, no. 4, pp. 371-380, 2018. 